\title{
Thermodynamical properties of protein kinase with adenine inhibitors
}

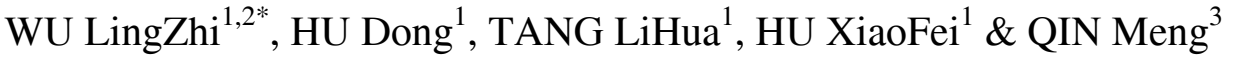 \\ ${ }^{1}$ School of Geography and Biological Information, Nanjing University of Posts and Telecommunications, Nanjing 210046, China; \\ ${ }^{2}$ State Key Laboratory of Bioelectronics, School of Biological Science and Medical Engineering, Southeast University, Nanjing 210096, China; \\ ${ }^{3}$ National Laboratory of Solid State Microstructure, Department of Physics, Nanjing University, Nanjing 210093, China
}

Received June 28, 2012; accepted September 5, 2012

\begin{abstract}
The protein-based molecular recognition of the adenine ring is essential to understand protein function and drug design as well. In this paper, a variety of the adenine-based inhibitors with modified groups of amino groups, nitrogen and oxygen atoms in the aromatic ring are designed, and the binding capability of these adenosine analogues with an aminoglycoside antibiotic kinase [APH(3')-IIIa] are investigated with activity assays and isothermal titration calorimetry (ITC) experiments. 1-aminoisoquinoline is one of the weakest substrates bound to APH(3')-IIIa with the lowest affinity (high $k_{i}$ and high $k_{d}$ ) and the smallest negative value of free energy change $(\Delta G)$ among the inhibitors tested. The binding process of adenine and 5-nitroisoquinoline to APH( $\left(3^{\prime}\right)$-IIIa is an enthalpy-driven event with unfavorable entropy, which is consistent with the energy change induced by the binding of ATP to the enzyme. However, the reverse is true for 1-aminoisoquinoline, 3-amino-5-nitrobenzisothiazole, 5-aminoisoquinoline binding to the enzyme because the entropy is more favorable and the enthalpy makes a lower contribution to the binding process. These results suggest that small changes of the adenine ring can lead to significant influence on the ability of these analogues to occupy the adenine-binding region of the enzyme, which can be the potential inhibitors as drug candidates against the bacterial resistance.
\end{abstract}

adenine inhibitor, aminoglycoside antibiotic kinase, ligand, recognition

Citation: Wu L Z, Hu D, Tang L H, et al. Thermodynamical properties of protein kinase with adenine inhibitors. Chin Sci Bull, 2013, 58: 68-73, doi: 10.1007/ s11434-012-5611-3

As a multifunctional nucleotide, adenosine 5'-triphosphate (ATP) plays critical roles across all forms of life. It is produced as an energy source to participate in the processes of cell signaling and protein synthesis [1-3]. In signal transduction pathways, molecular recognition of ATP with proteins has emerged as a subject of great interest. Recent studies on the protein kinase have demonstrated that most of the non-covalent interactions involved in the binding between proteins and the adenine ring are hydrogen-bonding, $\pi-\pi$ stacking, and cation- $\pi$ interactions [3-7]. The investigations on these specific non-covalent intermolecular interactions between protein and adenine are important in understanding the acting of protein functions and the design of new drugs. One of the enzymes which typifies the majority of these structural motifs is aminoglycoside 3 '-phosphotrans-

*Corresponding author (email: wulz@njupt.edu.cn) ferase (APH(3')-IIIa), a well-studied aminoglycoside phosphotransferase which is important for antibiotic drug resistance.

APH( $\left.3^{\prime}\right)$-IIIa is very promiscuous in its substrate selection, being able to phosphorylate the $3^{\prime}$ and 5'-hydroxyl functionalities of aminoglycosides dependent on the ATP hydrolysis [8-10]. Phosphorylated antibiotic cannot efficiently bind to its target, i.e. the bacterial ribosomal aminoacyl-tRNA site of the 16S RNA, which induces the resistance phenotype in organisms harboring the aminoglycoside phosphotransferases [11]. On the basis of the catalyzed modification reaction, these non-covalent interactions are critical for the recognition and modification of various antibiotics, i.e. the binding of nucleotides. Schematic of these non-specific interactions between APH(3')-IIIa and nucleotide ligands based on the crystal structure of the complex is shown in Figure 1. In detail, the interaction of $\pi-\pi$ stacking 


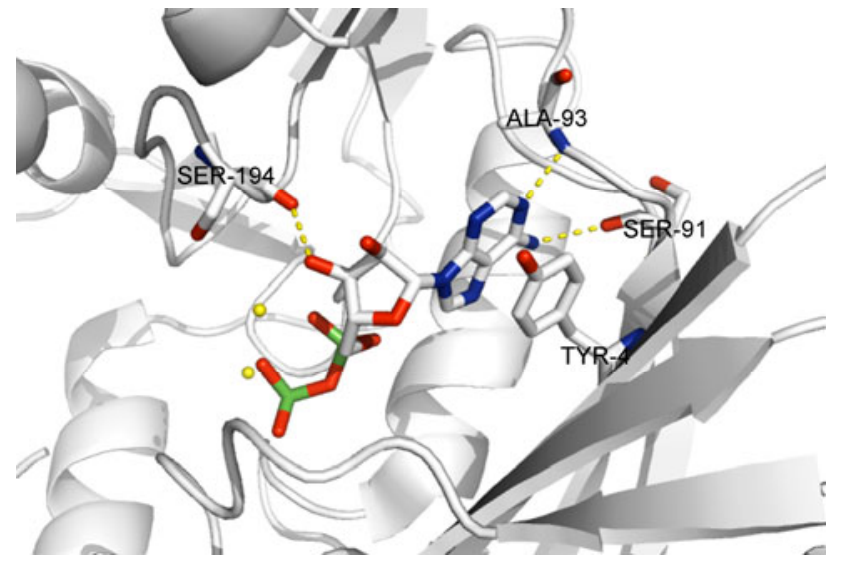

Figure 1 (Color online) Schematic representation of the non-specific interactions between $\mathrm{APH}\left(3^{\prime}\right)$-IIIa and nucleotide ligands based on the crystal structure of the complex.

occurs between aromatic ring of Tyr42 and adenine ring in ATP, and two hydrogen bonds locate between the N1 atom of the nucleotide and the backbone amide of Ala93, the N6 atom and the backbone carbonyl oxygen of Ser91, respectively. In addition, the ribose component interacts with the protein via a single hydrogen bond between the O3' oxygen of the sugar and the carbonyl oxygen of Ser194 [12-14]. Thus, the nitrogen and oxygen atoms in the adenine ring are vital for the recognition of the nucleotides with enzymes. It is likely that some of the adenine-based inhibitors can be designed to reverse the antibiotic resistance by taking advantage of the binding opportunities available in this deep, interaction-rich pocket of APHs.

In this work, five typical adenosine analogues are well designed to investigate the role of each group in adenosine ring binding to the aminoglycoside antibiotic kinase ( $\mathrm{APH}\left(3^{\prime}\right)$ IIIa), probed by activity assays and isothermal titration calorimetry experiments. As shown in Figure 2, these adenine analogues are typical structural motifs with different modified amino groups, nitrogen and oxygen atoms in the aromatic ring. The results indicate that the affinity of the 1 -aminoisoquinoline with three nitrogen atoms replaced by carbon in the adenine ring to $\mathrm{APH}\left(3^{\prime}\right)$-III is dramatically decreased with the lowest affinity (high $k_{i}$ and high $k_{d}$ ) and the smallest negative $\Delta G$ value among the five inhibitors. In contrast, the binding capability to APH(3')-IIIa is obviously enhanced for the cases of adenosine analogues 5-nitroisoquinoline and 3-amino-5-nitrobenzisothiazole due to the presence of more electronic groups, such as $\mathrm{O}-\mathrm{N}=\mathrm{O}$. In fact, the binding mechanisms of these adenosine analogues to enzyme are distinct from each other. Adenine and 5-nitroisoquinoline binding to $\mathrm{APH}\left(3^{\prime}\right)$-III is an enthalpy-driven event with unfavorable entropy, which is consistent with the energy changes with ATP binding to the enzyme. However, the bindings of 1-aminoisoquinoline, 3-amino-5-nitrobenzisothiazole, and 5-aminoisoquinoline to the enzyme are mainly driven by the entropy instead of enthalpy which

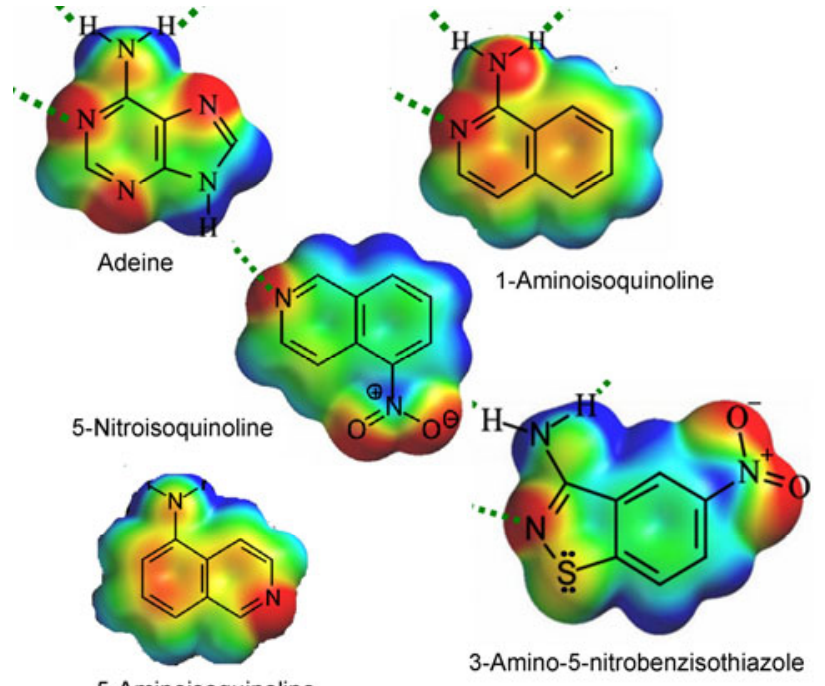

5-Aminoisoquinoline

Figure 2 (Color online) Electrostatic potential surface of some adenine analogues of adenine, 1-aminoisoquinoline, 5-nitroisoquinoline, 3-amino5-nitrobenzisothiazole and 5-aminoisoquinoline.

contributes very little to the binding. The results reveal that small changes in the structure of the adenine ring can lead to significant changes in the ability of these analogues to occupy the adenine-binding region of the enzyme. It is well known that the affinity of ATP as a cofactor to the enzyme is mainly determined by the interactions of the adenine ring, which is important for the recognition and modification of antibiotics with enzymes subsequently. Once cofactors fail to occupy the active region of enzymes priorly due to the presence of competitive inhibitors, the enzymes will hardly capture the antibiotics and modify them. Facing to the increasing resistance of antibiotics from modified enzymes, the investigation of these important interactions between protein kinase and typical adenosine analogues will shed light on drug design of the potential enzyme inhibitors to against the bacterial resistance.

\section{Materials and methods}

All adenine analogues were purchased from Sigma-Aldrich Co. (St. Louis, MO) with the highest purity commercially available, as shown in Figure 2. The enzymes of aminoglycoside antibiotic kinase were expressed and purified as before in our laboratory $[10,15]$. All other chemical reagents used in our experiment were of analytical grade without further purified. All buffer solutions were prepared with Milli-Q super purified water.

The catalytic activity of APH was determined in a coupled enzyme assay system based on the measurement of the decrease in NADH absorbance as described before [10,15]. Rate measurements were carried out by monitoring the decrease in absorbance at $340 \mathrm{~nm}$ on the Cary $50 \mathrm{UV}$-vis spectrophotometer (Varian Instruments, Palo Alto, CA) at 
room temperature.

Isothermal titration calorimetry experiments were performed at $25^{\circ} \mathrm{C}$ using a VP-ITC microcalorimeter from Microcal, Inc. (Northampton, MA). All measurements were carried out in $50 \mathrm{mM}$ Tris- $\mathrm{HCl}$ or HEPES buffers with 100 $\mathrm{mM} \mathrm{NaCl}$ at $\mathrm{pH} 7.5$, which also contained $10 \%(\mathrm{v} / \mathrm{v})$ DMSO to dissolve the ligands. The enzyme stock was dialyzed extensively against buffer, and ligand solutions were prepared in the final dialyzate. Both enzyme and ligand solutions were degassed under vacuum for $15 \mathrm{~min}$ at $20^{\circ} \mathrm{C}$. Titrations consisted of 29 injections of $10 \mu \mathrm{L}$ and were separated by $360 \mathrm{~s}$. Cell stirring speed was $300 \mathrm{r} / \mathrm{min}$. For each titration, the enzyme concentration was $20 \mu \mathrm{M}$ in the sample cell, and the inhibitor concentration was from 0.3 to 3 $\mathrm{mM}$ in the injection syringe. To obtain reliable dissociation constants, the $c$-value, a parameter obtained by the multiplication of the association constant and the total concentration of ligand binding sites [16], was kept between 10 and 100 for all titrations. Considering the affinity of 5-nitroisoquinoline with enzyme, the tertiary titration of adenine inhibitors into enzyme with tobramycin was repeated at the same conditions to accurate determine the competition of different ligands. The enzyme activity of APH( $\left.3^{\prime}\right)$-III was tested in the presence of osmotic solvent of DMSO, and no loss of activity was detected at the DMSO concentration less than $20 \%(\mathrm{v} / \mathrm{v})$. Control runs were performed by titrating ligands into buffer without enzyme, and the resulting reference signal was subtracted from the corresponding experimental data.

Thermodynamic parameters $n$ (stoichiometry), $k_{d}$ (dissociation constant), and $\Delta H$ (enthalpy change) were obtained by nonlinear least-squares fitting of the experimental data except 5-aminoisoquinoline using a single-site binding model of Origin software package provided by the instrument. The free energy of binding $(\Delta G)$ and entropy change $(\Delta S)$ were obtained using

$$
\begin{gathered}
\Delta G=-R T \ln K_{a}, \\
\Delta G=\Delta H-T \Delta S .
\end{gathered}
$$

The intrinsic enthalpy of binding was determined by the simultaneous solutions of

$$
\begin{gathered}
\Delta H_{\mathrm{abs} 1}=\Delta H_{\mathrm{int}}+\Delta H_{\text {ion } 1} \Delta n, \\
\Delta H_{\mathrm{abs} 2}=\Delta H_{\mathrm{int}}+\Delta H_{\mathrm{ion} 2} \Delta n .
\end{gathered}
$$

Here, the subscripts " 1 " and " 2 " refer to different buffers; $\Delta H_{\text {abs }}$ is the observed enthalpy change upon binding; $\Delta H_{\text {ion }}$ is the heat of ionization of the buffer; and $\Delta n$ is the net uptake of protons by the buffer upon complex formation $[17,18]$. The heat of ionization for HEPES is $+21.01 \mathrm{~kJ} / \mathrm{mol}(4.87$ $\mathrm{kcal} / \mathrm{mol})$ and that for Tris- $\mathrm{HCl}$ is $+49.75 \mathrm{~kJ} / \mathrm{mol}(11.7$ $\mathrm{kcal} / \mathrm{mol})$ [19].

\section{Results}

The thermodynamic properties for the binding between APH(3')-IIIa and several adenine inhibitors were studied by ITC. In detail, thermodynamic data for the formation of the binary enzyme-inhibitor complexes are present in Table 1 and Figure 3. It is shown that the binding constants and thermodynamic parameters are clearly different although these adenosine analogues have similar structures, processed with the adenine ring and similar size.

In the case of 1-aminoisoquinoline, three nitrogen atoms with negative and positive charge densities in adenine are substituted by carbon in the adenine ring. Although the inhibition constant is close to that of adenine, the association constant is decreased by $\sim 5$ fold. This result indicates that the electrostatic potential from these nitrogen atoms in the

\begin{tabular}{|c|c|c|c|c|c|c|c|}
\hline & Buffer & $k_{i}(\mu \mathrm{M})$ & $k_{d}(\mu \mathrm{M})$ & $\Delta H(\mathrm{kcal} / \mathrm{mol})$ & $T \Delta S(\mathrm{kcal} / \mathrm{mol})$ & $\Delta G(\mathrm{kcal} / \mathrm{mol})$ & $\Delta n$ \\
\hline \multirow{2}{*}{ Adenine } & Tris & \multirow{2}{*}{$50 \pm 8.0$} & 13.6 & -6.98 & +0.37 & -6.61 & \multirow{2}{*}{-0.001} \\
\hline & HEPES & & 16.7 & -6.97 & +0.48 & -6.49 & \\
\hline \multirow{2}{*}{ 1-Aminoisoquinoline } & Tris & \multirow{2}{*}{$60 \pm 9.5$} & 74.6 & -0.755 & -4.85 & -5.61 & \multirow{2}{*}{0.002} \\
\hline & HEPES & & 89.3 & -0.77 & -4.73 & -5.50 & \\
\hline 5-Nitroisoquinoline & Tris & $9.72 \pm 4.5$ & 2.85 & -8.62 & +1.08 & -7.54 & 1.007 \\
\hline \multirow{2}{*}{ 3-Amino-5-nitrobenzisothiazole } & Tris & \multirow{2}{*}{$5.8 \pm 1.5$} & 3.85 & -5.88 & -1.48 & -7.36 & \multirow{2}{*}{-0.635} \\
\hline & HEPES & & 4.38 & -1.54 & -5.74 & -7.28 & \\
\hline \multirow{2}{*}{ 5-Aminoisoquinoline } & Tris & \multirow{2}{*}{$35.4 \pm 7.5$} & 1.13 & -0.44 & -7.64 & -8.08 & \multirow{2}{*}{0.011} \\
\hline & HEPES & & 1.43 & -0.52 & -7.42 & -7.94 & \\
\hline ATP* & & & 11.0 & -17.5 & +10.6 & -6.88 & \\
\hline
\end{tabular}
adenine ring prefers the binding interactions of enzyme with analogues. However, for 5-aminoisoquinoline, in which the

Table 1 Thermodynamics parameters for adenine inhibitor binding to $\mathrm{APH}\left(3^{\prime}\right)-\mathrm{III}$ at $\mathrm{pH} 7.5^{\text {a) }}$

a) Fitting errors: $k_{d}<12 \%, \Delta H<10 \%, \Delta n<0.1 \%$. *, The value of ATP binding to APH(3')-IIIa were in $50 \mathrm{mM} \mathrm{HEPES-NaOH}$ at $\mathrm{pH} 7.5,40 \mathrm{mM} \mathrm{KCl,} \mathrm{and}$ $10 \mathrm{mM} \mathrm{MgCl}_{2}$ using ITC at $303 \mathrm{~K}$ as described in [5]. 


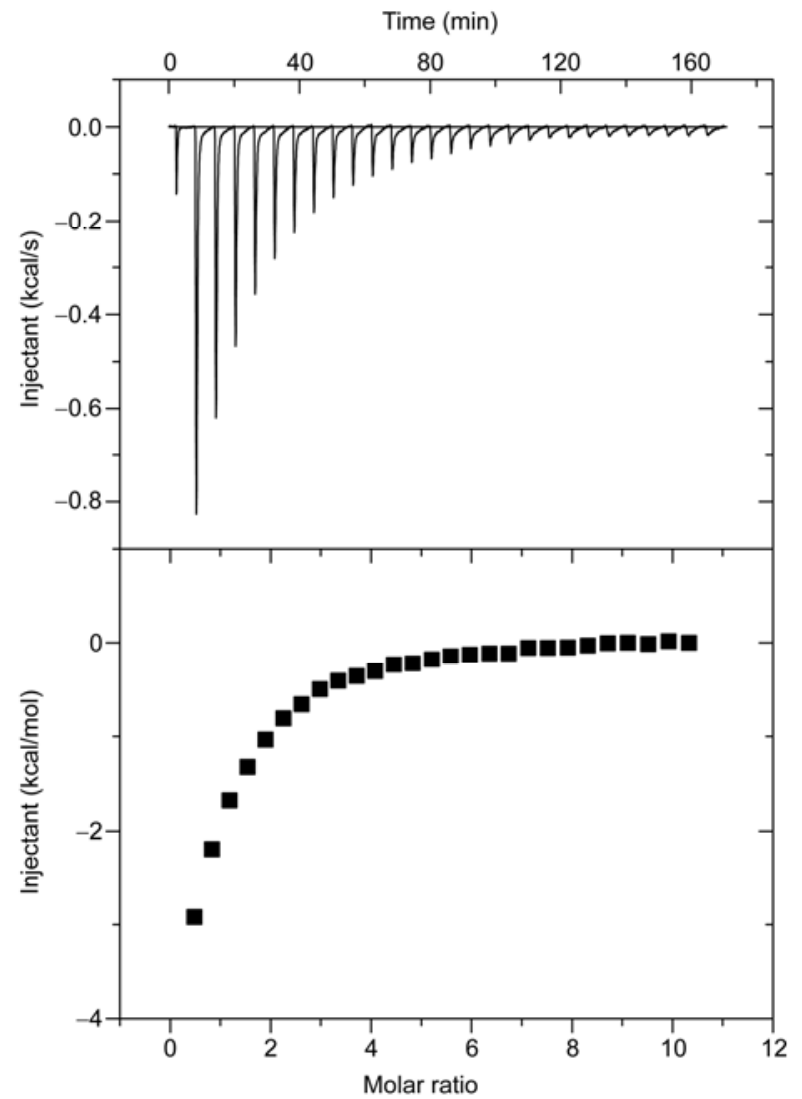

Figure 3 ITC profiles of adenine titration into APH( $\left.3^{\prime}\right)$-III in Tris- $\mathrm{HCl}$ buffer.

$\mathrm{N} 1$ atom is just moved to N5 position of 1-aminoisoquinoline, the association constant is increased by $\sim 80$ fold although the inhibition constant is only decreased by one-fold. The significant change of affinity constant indicates that the positive electron potential in N5 position is important for the binding of adenine to enzyme. With regard to other two adenosine analogues, i.e. 5-nitroisoquinoline and 3-amino5-nitrobenzisothiazole, they have the similar structures except an additional sulfur atom and an amino group in 3amino-5-nitrobenzisothiazole. They are the effective inhibitors (lower $k_{i}$ ) with higher affinity to $\mathrm{APH}\left(3^{\prime}\right)$-IIIa. It is found that the dissociation constant in ITC experiments is well correlated to the inhibition constants observed in kinetic assay; for example, the values of $k_{d}$ decrease with the decreasing $k_{i}$. In fact, there is an exception for the case of 5 -aminoisoquinoline with a larger value of $k_{i}$ but a smaller value of $k_{d}$, which may be caused by the positive electron potential in the N5 position of adenien ring. The affinity analyses from the adenine ring with different groups reveal that minor modifications on the adenine ring can lead to a significant change in the binding capability to enzymes.

In fact, such an effect can also be derived from the change of their thermodynamic properties. The binding process of adenine analogues to APH( $\left.3^{\prime}\right)$-IIIa exhibits large exothermic heat associating with negative change of free energy $\Delta G$ values. The window of negative $\Delta G$ values is as narrow as $2.58 \mathrm{kcal} / \mathrm{mol}$ (from -5.50 to $-8.08 \mathrm{kcal} / \mathrm{mol}$ ). In addition, the change of enthalpy $\Delta H$ values varies from -0.44 to $-15.5 \mathrm{kcal} / \mathrm{mol}$, and the contribution of entropy to the free energy $(-T \Delta S)$ varies from -7.64 to $8.52 \mathrm{kcal} / \mathrm{mol}$. The results show that 1-aminoisoquinoline is one of the weakest substrates binding to APH( $\left.3^{\prime}\right)$-IIIa with the lowest value of affinity (high $k_{i}$ and $k_{d}$ ) and the smallest change of negative $\Delta G$ value among the five inhibitors tested in this work. As shown in Table 1 and Figure 4, the binding process of adenine and 5-nitroisoquinoline to APH(3')-IIIa is an enthalpydriven event with unfavorable entropy, which consists well with the energy changes in the binding process between ATP and the enzyme [15]. However, for the case of 1aminoisoquinoline, 3-amino-5-nitrobenzisothiazole, and 5aminoisoquinoline, it is quite different that the entropy is more favorable and the contribution of enthalpy is extremely small.

Additionally, the tertiary titrations of some adenine analogues with high affinity to APH(3')-IIIa were also performed in the presence of tobromycin. For 5-nitroisoquinoline, the association constants and the enthalpic contribution do not change too much (less than $30 \%$ ), but the disfavorable entropic contribution is decreased by $1-2$ folds in the presence of antibiotics. For the case of 3-amino-5-nitrobenzisothiazole, the accurate free energy changes can be hardly measured in the presence of tobramycin due to the extremely low association constant. Tobramycin is a competitive inhibitor of APH(3')-IIIa, which lacks the $3^{\prime}$-hydroxyl group of kanamycin $\mathrm{B}$ and cannot be modified by the enzyme [18]. The binding of tobramycin to APH(3')-IIIa is much tighter than these adenine analogues $\left(k_{d}=0.8 \mu \mathrm{M}\right)$. Thus, the favorable enthalpy compensates the entropic penalty yielding more negative $\Delta G$ values. The large effect of tobramycin on the binding process of adenine inhibitor to enzymes indicates that some active sites for antibiotics on enzyme may be occupied or changed in the association of enzyme and adenine analogues.

As shown in Table 1, all titrations are performed in two different buffers with different heat of ionization (Tris, 11.7 $\mathrm{kcal} / \mathrm{mol}$ vs. HEPES, $4.87 \mathrm{kcal} / \mathrm{mol}$ ) to determine protonation/deprotonation of functional groups involved in the binding interaction. This observation shows that $\Delta n$ values are zero during the formation of APH( $\left.3^{\prime}\right)$-IIIa with adenine, 1-aminoisoquinoline and 5-aminoisoquinoline although these analogues are with the $-\mathrm{NH}_{2}$ groups on the adenine ring. This may be a proper balance between the electrostatic density on the aromatic system and the additional protonation/ deprotonation accompanied in the enzyme side chains. For 5-nitroisoquinoline and 3-amino-5-nitrobenzisothiazole, the observed $\Delta n$ values are 1.007 and -0.635 , respectively. If nitrogen atom and $\mathrm{O}-\mathrm{N}=\mathrm{O}$ group in adenine ring are responsible for the protonation of 5-nitroisoquinoline, additional fraction of $-\mathrm{NH}_{2}$ group and sulfur atom in 3-amino- 

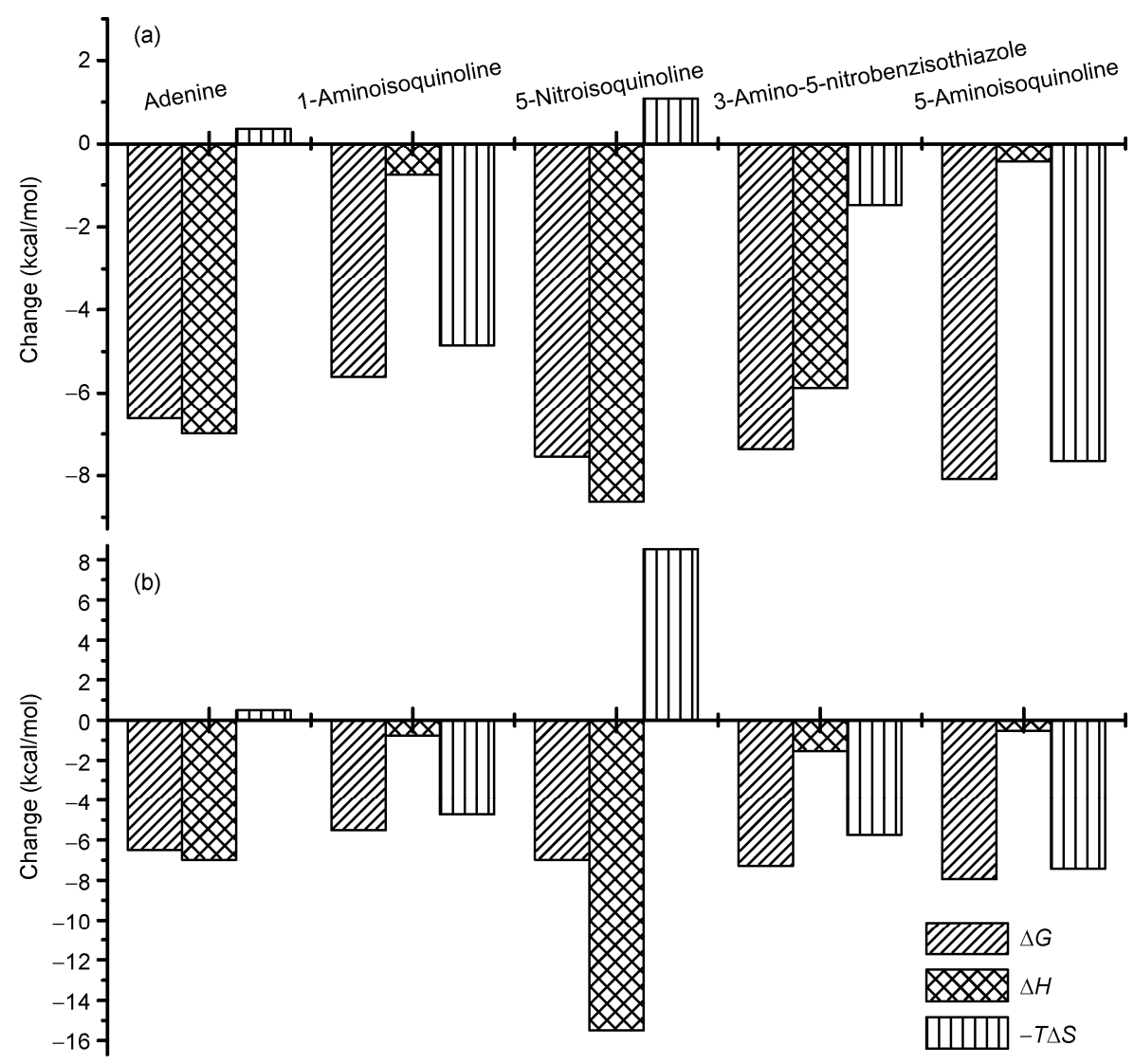

Figure 4 The changes of the free energy $(\Delta G)$, enthalpy $(\Delta H)$ and entropy $(-T \Delta S)$ of different adenine analogues binding to $\mathrm{APH}\left(3^{\prime}\right)-\mathrm{III}$ in Tris- $\mathrm{HCl}(\mathrm{a})$ and HEPES buffers (b).

5-nitrobenzisothiazole will be responsible for deprotonation of some groups in the complexes. Similarly, the contribution from the ionization of enzyme side chain to the observed heat change of the complex formation cannot be excluded although it is different in the presence of adenine analogues. Note that the interpretation of changes in enthalpy and entropy due to the modification on the adenine ligands may be complicated. The contribution of solvent, the compensation of enthalpy and entropy, and the effects of cooperative hydrogen bonding are all critical to the binding process. However, the change of the free energy due to a single substituent on the adenine inhibitors reveals the molecular determinants of affinity and specificity for nucleotide ligands binding to APH(3')-IIIa.

\section{Discussion}

As mentioned above, the binding of ATP to APH( $\left(3^{\prime}\right)$-IIIa is enthalpically driven and exhibits a strongly disfavored entropic contribution. The negative $\Delta H$ values are associated with the favorable binding contacts, such as polar interactions, electrostatic forces, van der Waals forces, and hydrogen bonds between the adenine ring and active sites of APH(3')-IIIa [12-14]. As shown in Figure 1, these interac- tions consist of the $\pi-\pi$ stacking with aromatic ring of Tyr42, the hydrogen bonds between the nucleotide and the backbone amide of Ala93 and one between the N6 atom and the backbone carbonyl oxygen of Ser91. Additionally, the ribose component interacts with the protein via a single hydrogen bond between the O3' oxygen of the sugar and the carbonyl oxygen of Ser194 [18-20]. So these amino groups, nitrogen and oxygen atoms in the aromatic ring are important for the recognition of the nucleotides with enzymes. The addition and substitution of some groups in the adenine analogues can disrupt the contacts between them, and lead to the associated energy changes. As shown in Table 1 , the enthalpic and entropic contributions are quite different although the free energies of adenine and 5-nitroisoquinoline binding to APH $\left(3^{\prime}\right)$-III are close to that of ATP with enzymes. In contrast to the case of ATP, the favorable enthalpy and disfavorable entropy both decreased during the formation of enzyme and adenine analogues, and thus the final energy does not change too much. The structure of adenine is close to ATP with $\mathrm{N} 1$ atom and $\mathrm{NH}_{2}$ in $\mathrm{N} 6$ of adenine ring. However, 5-nitroisoquinoline without amino group behaves more enthalpically. This may be responsible to the $\mathrm{N} 1$ and $\mathrm{O}-\mathrm{N}=\mathrm{O}$ group in adenine ring. Especially the $\mathrm{O}-\mathrm{N}=\mathrm{O}$ group contributes to the proton transfer during the interactions. The enthalpical contributions are less favorable 
and the entropical contributions become more favorable for the binding of 1-aminoisoquinoline, 3-amino-5-nitrobenzisothiazole, 5-aminoisoquinoline with the enzyme. These results suggest that the electrostatic interactions are diminished and the increased hydrophobic interactions between enzyme and ligands play a prominent role in these cases. However, it is somewhat exceptional for 3-amino-5-nitrobenzisothiazole with more electronic groups, such as $\mathrm{NH}_{2}$, $\mathrm{O}-\mathrm{N}=\mathrm{O}$ groups and nitrogen and sulfur atoms. Probably, the aromatic stacking interactions between $\mathrm{APH}\left(3^{\prime}\right)$-IIIa and adenine ring can supply to most of the total nucleotidebinding energy [19-21]. Certainly the minor change in the structure of the enzyme and adenine ring can not only lead to the disruption of a single contact but also influence the nature of remaining contact. And the ability of the enzyme to interact with the modified nucleobases will be attributed to the global changes in the thermodynamic parameters.

Overall, considering the importance of ATP as a cofactor of the enzymatic modification on the drug of antibiotics, several adenine analogues are constructed and characterized with the model system of APH(3')-IIIa [1-3,8-10]. The studies permit us to determine individual contribution of selected amino groups, nitrogen and oxygen atoms in the aromatic ring of the ligands to the global thermodynamic properties of the enzyme-ligand complex. Results described in this paper show that the recognition of APH with adenine analogues involves a complex pattern associated with the favorable binding contacts, and protonation and deprotonation between the adenine ring and active sites of $\mathrm{APH}\left(3^{\prime}\right)$ IIIa under the given experiment conditions. These findings highlight that small changes in the structure of the adenine ring can lead to significant changes in the ability of these analogues to occupy the adenine-binding region of the enzyme. Because of the prevalence of the unique aminoglycoside modifying enzymes, our work based on the molecular recognition of enzymes with adenine analogues provides a useful way for the design of enzyme inhibitors that have the potential to reverse aminoglycoside antibiotic.

This work was supported by the National Natural Science Foundation of China (61101056), the Open Project of State Key Laboratory of Bioelectronics of Southeast University (2011E14), the China Postdoctoral Science Foundation (20110491339) and the Scientific Research Foundation of Nanjing University of Posts and Telecommunications (NY210082).

1 Schulz G E. Binding of nucleotides by proteins. Curr Opin Struct Biol, 1992, 2: 61-67

2 Vetter I R, Wittinghofer A. Nucleoside triphosphate-binding proteins: Different scaffolds to achieve phosphoryl transfer. Quart Rev Biophys, 1999, 32: 1-56

3 Kryger G, Silman I, Sussman J L. Structure of acetylcholinesterase complexed with E2020 (Aricept): Implications for the design of new anti-Alzheimer drugs. Structure, 1999, 7: 297-307

4 Meyer E A, Castellano R K, Diederich F. Interactions with aromatic rings in chemical and biological recognition. Angew Chem Int Ed, 2003, 42: 1210-1250

5 Burk D L, Hon W C, Leung A K, et al. Structural analyses of nucleotide binding to an aminoglycoside phosphotransferase. Biochemistry, 2001, 40: 8756-8764

6 Boehr D D, Farley A R, Wright G D, et al. Analysis of the $\pi-\pi$ Stacking Interactions between the aminoglycoside antibiotic kinase APH(3')-IIIa and its nucleotide ligands. Chem Biol, 2002, 9: 1209-1217

7 Kinoshita T, Sekiguchi Y, Fukada H, et al. A detailed thermodynamic profile of cyclopentyl and isopropyl derivatives binding to CK2 kinase. Mol Cell Biochem, 2011, 356: 97-105

8 McKay G A, Wright G D. Kinetic mechanism of aminoglycoside phosphotransferase type IIIa evidence for a theorell-chance mechanism. J Biol Chem, 1995, 270: 24686-24692

9 Fong D H, Berghuis A M. Substrate promiscuity of an aminoglycoside antibiotic resistance enzyme via target mimicry. EMBO J, 2002, 21: 2323-2331

10 McKay G A, Thompson P R, Wright G D. Broad spectrum aminoglycoside phosphotransferase type III from enterococcus: Overexpression, purification, and substrate specificity. Biochemistry, 1994, 33: 6936-6944

11 Llano-Sotelo B, Azucena E F Jr, Kotra L P, et al. Aminoglycosides modified by resistance enzymes display diminished binding to the bacterial ribosomal aminoacyl-tRNA site. Chem Biol, 2002, 9: 455-463

12 Boehr D D, Lane W S, Wright G D. Active site labeling of the gentamicin resistance enzyme $\mathrm{AAC}\left(6^{\prime}\right)-\mathrm{APH}\left(2^{\prime \prime}\right)$ by the lipid kinase inhibitor wortmannin. Chem Biol, 2001, 8: 791-800

13 Burk D L, Berghuis A M. Protein kinase inhibitors and antibiotic resistance. Pharmacol Ther, 2002, 93: 283-292

14 Burk D L, Hon W C, Leung A K W, et al. Structural analyses of nucleotide binding to an aminoglycoside phosphotransferase. Biochemistry, 2001, 40: 8756-8764

15 Thompson P R, Boehr D D, Berghuis A M, et al. Mechanism of aminoglycoside antibiotic kinase APH(3')-IIIa: Role of the nucleotide positioning loop. Biochemistry, 2002, 41: 7001-7007

16 Wu L Z, Serpersu E H. Deciphering interactions of the aminoglycoside phosphotransferase(3')-IIIa with its ligands. Biopolymers, 2009, 91: 801-809

17 Wiseman T, Williston S, Brandts J F, et al. Rapid measurement of binding constants and heats of binding using a new titration calorimeter. Anal Biochem, 1989, 179: 131-137

18 Wright E, Serpersu E H. Enzyme-substrate Interactions with an antibiotic resistance enzyme: Aminoglycoside nucleotidyltransferase(2")Ia characterized by kinetic and thermodynamic methods. Biochemistry, 2005, 44: 11581-11591

19 Özen C, Serpersu E H. Thermodynamics of aminoglycoside binding to aminoglycoside-3'-phosphotransferase IIIa studied by isothermal titration calorimetry. Biochemistry, 2004, 43: 14667-14675

20 Goldberg R N, Kishore N, Lennen R M. Thermodynamic quantities for the ionization reactions of buffers. J Phys Chem Ref Data, 2002, 31: 231-370

21 Boehr D D, Farley A R, LaRonde F J, et al. Establishing the principles of recognition in the adenine-binding region of an aminoglycoside antibiotic kinase [APH(3')-IIIa]. Biochemistry, 2005, 44: 1244512453

22 Fong D H, Berghuis A M. Structural basis of APH(3')-IIIa-mediated resistance to N1-substituted aminoglycoside antibiotics. Antimicrob Agents Ch, 2009, 53: 3049-3055

Open Access This article is distributed under the terms of the Creative Commons Attribution License which permits any use, distribution, and reproduction in any medium, provided the original author(s) and source are credited. 